\title{
Evaluation of Antioxidant Activity in Vitro of Polyphenols from Toona Sinensis Seeds Using RSM Optimized Conditions
}

\author{
Wanzhong $\mathrm{Li}^{*}$, Bing Liu, Yan Lu, Wenxian Jiang and Fei Wang \\ ${ }^{1}$ Pharmacy college, Weifang Medical University, China \\ ${ }^{*}$ Corresponding author
}

Keywords: Toona Sinensis Seeds, Polyphenols, Response surface methodology, Antioxidant activity.

\begin{abstract}
The aim was to establish a method for extracting polyphenols from Toona sinensi Seeds (PTSS) and evaluate antioxidant properties. The study selected the polyphenols extraction yield as processing parameters and volume fraction, ratio of liquor to material, extraction time and extraction power as independent variables and was carried out by response surface methodology(RSM).The optimal ultrasound-assisted extraction (UAE)yield of $2.64 \mathrm{mg} / \mathrm{g}$ was obtained with a volume fraction of 43\%,a ratio of liquor to material of 19:1,an extraction time of 13 min and the use of $211 \mathrm{w}$ ultrasonic power. PTSS has a certain antioxidant capacity in vitro. The UAE is stable and reliable. The results indicate that Toona Sinensis Seeds is a medical and edible dual purpose material with potential antioxidant properties.
\end{abstract}

\section{Introduction}

Toona sinensisis extensively distributed in China. Its chemical compositions mainly include polyphenols, saponins, alkaloids[1].PTSS have antioxidant effects[2],lowers blood sugar levels[3],anti-inflammatory[4], improve function of nerve cells and endothelial cells[5-6], et al. Polyphenols are antioxidant phytochemicals and own both singlet oxygen quenching activity and radical scavenging activity[7].Although studies demonstrated Toona Sinensis Seeds contains polyphenols[1], little is known about antioxidant activities of PTSS.

UAE is characterized by short extraction time, high efficiency, simple operation and low price[8].The response surface function and contour are analyzed in order to study the relationship between factors, as well factor and response surface. RSM is adopted for UAE of PTSS. It makes a study on the in vitro antioxidant activity of PTSS, and lays a foundation for exploitation of Toona Sinensis Seeds and its activity evaluation.

\section{Instrument and Reagent}

SCIENTZ-IID ultrasonic cell crusher (Ningbo Scientz Biotechnology Co., Ltd.), UV8000 UV-visible spectrophotometer (Shanghai Precision Instrument Co., Ltd.), Electronic analytical balance EL204 (Mettler-Toledo Instruments Shanghai Co., Ltd.).

Toona Sinensis Seeds (Jinan Shengke Technology Development Co., Ltd.); Gallic acid (National Institutes for Food and Drug Control), Total antioxidant capability (T-AOC) and superoxide anion kits (Nanjing Jiancheng Bioengineering Institute). DPPH (St. Louis, MO, USA).

\section{Methods}

Detection of polyphenols content [9]. Different volumes(0, 20,50,100, 150, 200, 250 $\mu \mathrm{L})$ of garlic acid at $0.2 \mathrm{mg} / \mathrm{mL}$ were measured and added to water to $250 \mu \mathrm{L}$.Exactly $0.4 \mathrm{~mL}$ of folin-phenol reagent was added into the solution and placed for $1 \mathrm{~min}$.Sodium carbonate solution $(0.4 \mathrm{~mL}, 10 \%)$ 
and water $(4.0 \mathrm{~mL})$ were added, and mixture was shaken. The absorbance was detected at $760 \mathrm{~nm}$ after 20min.With the content of garlic acid control as horizontal coordinate and the absorbance as vertical coordinate, the standard curve was drawn.

Ultrasound-assisted Extraction. Single factor investigation. $2.0 \mathrm{~g}$ seeds are weighed and the effects on extracting polyphenols of ethanol volume fraction(30,40, 50, 60, 70\%), ratio of liquor to material $(10: 1,20: 1,30: 1,40: 1,50: 1)$, extraction time(5, 10, 15, 20, 25min) and extraction power(100, 150, 200, 250, 300w) were investigated by parallel laboratory tests.

RSM for optimization of extraction process. Based on single factor, take three levels of each factor, measurement would be content of polyphenols. Design-Expert software is adopted to establish a mathematical model and optimize UAE parameters.

Antioxidant activity in vitro. Determination of total antioxidant capacity. T-AOCkit and microplate were used for quantitative assay. Polyphenols were dissolved with ethanol, with concentration $(\mathrm{mg} / \mathrm{mL})$ as horizontal coordinate and absorbance as vertical coordinate, Vc was used for positive control.

Determination of DPPH radical scavenging. DPPH was dissolved with ethanol and diluted with ethanol to be $0.2 \mathrm{mmol} / \mathrm{L}[10]$.During the reaction, DPPH solution of the same volume was added and placed away from light for $15 \mathrm{~min}$. The absorbance was detected at $515 \mathrm{~nm}$.

Determination of PTSS capability of scavenging $\mathrm{O}^{2-}$ The anti-superoxide anion radical kit and xanthine oxidase method were used with Vc as standard to determine the capability of scavenging superoxide anion radicals.

Statistical Analysis. Experiments were conducted in triplicate and statistical analyses were carried out according to SPSS 15.0. Differences were considered significant with $\mathrm{p}<0.05$.

\section{Results}

Establishment of standard curve. It shows a good linear correlation in the concentration range of garlic acid from 0 to $2.0 \mathrm{mg} / \mathrm{ml}$, the regression equation: $\mathrm{y}=0.513 \mathrm{x}+0.0031, \mathrm{r}=0.9996$.

Single Factor Investigation. Influence of ethanol volume fraction on yield of PTSS. With the increase of ethanol volume fraction, the extraction yield of PTSS firstly increases and then decreases. When volume fraction is $50 \%$, extraction yield reached the maximum.

Influence of ratio of liquor to material on yield of PTSS. PTSS extraction yield firstly increases and then decreases with the increase of ratio of liquor to material. It was observed that the extraction yield of PTSS was maximum at a liquor to material ratio of 20:1 (v/w).

Influence of extraction time on yield of PTSS. PTSS extraction yield firstly increases and then decreases with the increase of extraction time. When the extraction time is $15 \mathrm{~min}$, PTSS extraction yield is the maximum.

Influence of extraction power on yield of PTSS. With the increase of ultrasonic power, PTSS extraction yield firstly increases and then decreases. When the power is 200W, PTSS extraction reached the maximum.

RSM for the optimization of extraction process. Modeling and fitting the model using RSM. The design and response data for PTSS yield are presented in Table 1, 2.The regression coefficients of the intercept, linear, quadratic and interaction terms of the model are given in Table 3. 
Table 1. Variables and their levels

\begin{tabular}{cccc}
\hline & \multicolumn{3}{c}{ Levels } \\
\cline { 2 - 4 } Variables & -1 & 0 & 1 \\
\hline Ethanol volume fraction(\%) & 40 & 50 & 60 \\
\hline Ratio of liquor to material & $10: 1$ & $20: 1$ & $30: 1$ \\
\hline Extraction time(min) & 10 & 15 & 20 \\
\hline Extraction power(w) & 150 & 200 & 250 \\
\hline
\end{tabular}

Table 2. Experimental and predicted values of PTSS

\begin{tabular}{cccccc}
\hline Run & A & B & C & D & Yield \\
\hline 1 & 0 & 1 & 0 & 1 & 2.258 \\
2 & 0 & 0 & 0 & 0 & 2.597 \\
3 & 1 & 0 & 0 & 1 & 2.068 \\
4 & 0 & 0 & 0 & 0 & 2.600 \\
5 & -1 & 0 & 0 & 1 & 2.279 \\
6 & 1 & 0 & 0 & -1 & 1.702 \\
7 & 0 & -1 & 0 & 1 & 1.638 \\
8 & 1 & 0 & 1 & 0 & 2.284 \\
9 & 0 & 0 & 0 & 0 & 2.583 \\
10 & 0 & 0 & -1 & 1 & 2.301 \\
11 & 0 & -1 & 0 & -1 & 1.691 \\
12 & 0 & 1 & -1 & 0 & 1.961 \\
13 & -1 & -1 & 0 & 0 & 2.103 \\
14 & 0 & 0 & 0 & 0 & 2.562 \\
15 & 0 & 1 & 1 & 0 & 2.144 \\
16 & 0 & 0 & 0 & 0 & 2.632 \\
17 & 0 & -1 & 1 & 0 & 1.790 \\
18 & -1 & 0 & 0 & -1 & 1.965 \\
19 & 1 & 1 & 0 & 0 & 2.091 \\
20 & 0 & 0 & -1 & -1 & 1.371 \\
21 & -1 & 0 & 1 & 0 & 2.104 \\
22 & -1 & 1 & 0 & 0 & 2.301 \\
23 & 1 & -1 & 0 & 0 & 1.834 \\
24 & 0 & 1 & 0 & -1 & 1.524 \\
25 & 1 & 0 & -1 & 0 & 1.808 \\
26 & 0 & 0 & 1 & 1 & 1.767 \\
27 & 0 & -1 & -1 & 0 & 2.078 \\
28 & 0 & 0 & 1 & -1 & 2.004 \\
29 & -1 & 0 & -1 & 0 & 2.465 \\
\hline
\end{tabular}


Table 3. Analysis of variance for the regression equation

\begin{tabular}{|c|c|c|c|c|c|c|}
\hline source & sum of squares & df & mean square & F value & $\mathrm{p}$ value & significance \\
\hline model & 3.32 & 14 & 0.24 & 152.55 & $<0.0001$ & $* * *$ \\
\hline A-Ethanol volume & 0.17 & 1 & 0.17 & 109.61 & $<0.0001$ & $* * *$ \\
\hline C-Extraction time & 0.000997 & 1 & 0.000997 & 0.64 & 0.4364 & \\
\hline D-Extraction power & 0.35 & 1 & 0.35 & 226.37 & $<0.0001$ & $* * *$ \\
\hline $\mathrm{AB}$ & 0.000870 & 1 & 0.000870 & 0.56 & 0.4666 & \\
\hline $\mathrm{AC}$ & 0.18 & 1 & 0.18 & 112.62 & $<0.0001$ & $* * *$ \\
\hline $\mathrm{AD}$ & 0.000676 & 1 & 0.000676 & 0.43 & 0.5202 & \\
\hline $\mathrm{BC}$ & 0.055 & 1 & 0.055 & 35.6 & $<0.0001$ & $* * *$ \\
\hline $\mathrm{BD}$ & 0.15 & 1 & 0.15 & 99.61 & $<0.0001$ & $* * *$ \\
\hline CD & 0.34 & 1 & 0.34 & 218.98 & $<0.0001$ & $* * *$ \\
\hline $\mathrm{A}^{\wedge} 2$ & 0.15 & 1 & 0.15 & 96.9 & $<0.0001$ & $* * *$ \\
\hline $\mathrm{B}^{\wedge} 2$ & 0.8 & 1 & 0.8 & 513.92 & $<0.0001$ & $* * *$ \\
\hline $\mathrm{C}^{\wedge} 2$ & 0.47 & 1 & 0.47 & 300.25 & $<0.0001$ & $* * *$ \\
\hline $\mathrm{D}^{\wedge} 2$ & 1.35 & 1 & 1.35 & 871.46 & $<0.0001$ & $* * *$ \\
\hline Residual & 0.022 & 14 & 0.00155 & & & \\
\hline Lack of Fit & 0.019 & 10 & 0.00191 & 2.92 & 0.157 & \\
\hline Pure Error & 0.00263 & 4 & 0.000656 & & & \\
\hline \multirow[t]{2}{*}{ Cor Total } & 3.34 & 28 & & & & \\
\hline & $\mathrm{R}^{2}=0.9935$ & & $\mathrm{R}^{2} \mathrm{AD}_{\mathrm{j}}=0$ & 9870 & & \\
\hline
\end{tabular}

It was shown quadratic parameters were highly significant at the level of $\mathrm{p}<0.01$, the order of extraction rate influenced by the factors is extraction power (D) $>$ volume fraction(A) $>$ ratio of liquor to material (B)>extraction time (C),the final predictive equation was obtained as follows, $\mathrm{Y}=2.59-0.12 \mathrm{~A}+0.095 \mathrm{~B}+0.009 \mathrm{C}+0.17 \mathrm{D}+0.015 \mathrm{AB}+0.21 \mathrm{AC}+0.013 \mathrm{AD}+0.12 \mathrm{BC}+0.2 \mathrm{BD}-0.29 \mathrm{CD}-0.1$ $5 \mathrm{~A}^{2}-0.35 \mathrm{~B}^{2}-0.27 \mathrm{C} 2-0.46 \mathrm{D}^{2}$

The regression equation $\mathrm{F}$ is $152.55, \mathrm{p}<0.0001$, indicating that the regression model is significant, correlation coefficient $\mathrm{R}^{2}=0.9935$,illustrate the model has good fitting, correlation of multiple correlation coefficient $\mathrm{R}^{2} \mathrm{Adj}=0.9870$, indicating the model can explain $98.70 \%$ of changes in response.

RSM Analysis. Three dimensional response surface profiles of multiple non-linear regression models were plotted. Contour and response surface graph are drawn according to the regression equation shown in Fig. 1.Each combination of AC, BC, BD, CD can affect the polyphenols extraction yield. When the response value reaches the extremes, the response value will decrease with the increase of factor. 

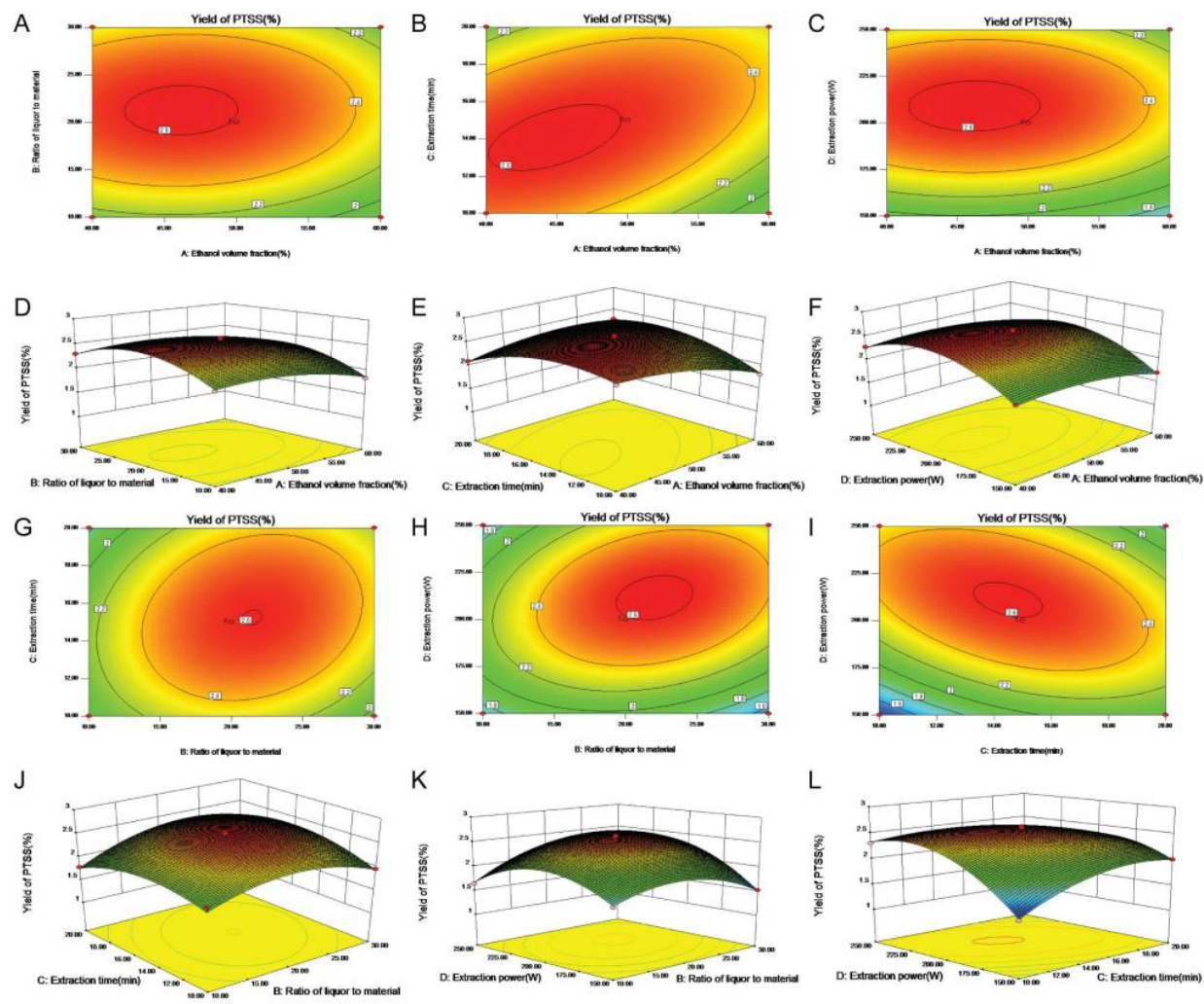

Fig. 1. Contour plots and response surface showing the effects of ethanol volume fraction, extraction time, ratio of liquor to material, extraction power on the PTSS extraction

The interaction between extraction time and power is oval in contour picture, and its response surface graph has steep surfaces, indicating that it has significant effect on PTSS yield. The interactions are oval in contour picture and response surface graphs have steep surfaces except for $\mathrm{AB}$ and $\mathrm{AD}$, indicating they have significant effect on PTSS yield.

Process Optimization. The conditions for UAE are as follows, volume fraction is 43\%,ratio of liquor to material is 19 ,extraction time is $13 \mathrm{~min}$, extraction power is $211 \mathrm{~W}$, polyphenols extraction yield is $2.64 \mathrm{mg} / \mathrm{g}$.

Antioxidant Activity in Vitro. Determination of total antioxidant capacity. The T-AOC increased with increase of concentration in the range of 0 to $1.5 \mathrm{mg} / \mathrm{mL}$. The trends that tend to level out by exceeding $1.5 \mathrm{mg} / \mathrm{mL}$ with the increasing of the concentration. It can provide a material base for efficiently separating and purifying natural antioxidants.

$\mathrm{DPPH}$ and $\mathrm{O}^{2-}$ Scavenging Method. The $\mathrm{IC}_{50}$ values of PTSS and ascorbic acid on DPPH radicals and superoxide anion radicals were detected shown in Table 4.The results indicates that PTSS can scavenge DPPH and $\mathrm{O}^{2-}$.

Table 4. $\mathrm{IC}_{50}$ of PTSS on DPPH and $\mathrm{O}^{2-}$

\begin{tabular}{ccc}
\hline Indexes & \multicolumn{2}{c}{$\mathrm{IC}_{50}$} \\
\hline & PTSS & $\mathrm{V}_{\mathrm{C}}$ \\
\hline $\mathrm{DPPH}(\mu \mathrm{g} / \mathrm{mL})$ & $16.19 \pm 1.28$ & $5.13 \pm 0.69$ \\
\hline $\mathrm{O}^{2-}(\mu \mathrm{g} / \mathrm{mL})$ & $95.47 \pm 2.61$ & $36.27 \pm 1.62$ \\
\hline
\end{tabular}

\section{Summary and Discussion}

The anti-oxygenation could be ascribed to phenolic compounds, such as phenolic acids, tannins, flavonoids and phenolic diterpenes[11].PTSS have potential antioxidant capability and scavenging effect in removing $\mathrm{DPPH}$ and $\mathrm{O}^{2-}$,it provides a basis for the discovery and rational use of plant-derived antioxidants. The study suggests the extracts are latent nutritional antioxidants. Further study is required to isolate and identify the antioxidant polyphenol compounds from ethanol extracts of PTSS. 


\section{Acknowledgments}

This work was supported by Shandong Science and Technology Research Project of TCM (2015227 and 2015231).

\section{References}

[1] Kakumu, A., Ninomiya, M., Efdi, M., Adfa, M., Hayashi, M., Tanaka, K., Koketsu, M, Phytochemical analysis and antileukemic activity of polyphenolic constituents of Toona sinensis, Bioorg Med Chem Lett, vol.24, pp. 4286-4290, 2014.

[2] XING Sha-sha, CHEN Chao, Study on the Antioxidation of Polyphenols from the Seeds of Toona sinensis (A. Juss) Roem in vitro, Journal ofAnhui Agri. Sci., vol.38, pp. 7285-7287, 2010.

[3] Xing Shasha, Chen Chao, Studies on hypoglycemic effects of total polyphenols from the seeds of toona sinensis, Chinese Journal of Herbal Pharmacology, vol.27, pp. 42-44, 2011.

[4] Yang Yanli, CHEN Chao, Effects of Total Polyphenols from Seeds of Toona Sinensis in Treating Adjuvant-induced Arthritis Rats, Chin JMAP, vol.29, pp. 1073-1077, 2012.

[5] Zhao Qiong, Sun Qianyun, Yang Qingxiong, Effects of an anticomplementary polyphenol from the seed of Toona sinensis on complement-injured SH-SY5Y cells, Chinese Pharmacological Bulletin, vol.27, pp. 1086-1090, 2011.

[6] Zhao Qiong, Sun Qianyun, Yang Qingxiong, Protective effects of a polyphenol from the seed of Toona sinensis onactivation and injury of endothelial cell induced by complement, Chinese Pharmacological Bulletin, vol.27, pp. 1401-1404, 2011.

[7] Al-Awwadi, N.,Azay, J.,Poucheret, P.,Cassanas, G.,Krosniak, M.,Auger, C.,Gasc, F.,Rouanet, J.-M.,Cros, G.,Teissedre, P.L., Redwine polyphenols alone or in association with ethanol prevent hypertension, cardiac hypertrophy, and production of reactive oxygen species in the insulin-resistant fructose-fed rat, J.Agric. Food Chem., vol.52, pp. 1008-1016, 2004.

[8] RQ. Jing, QP. Xiong, Y. Jing, Optimization of Ultrasonic-assisted Extraction of Polysaccharides from Fructus Mori by Response Surface Methodology, Natural Product Research and Development, vol.26, pp. 570, 2014.

[9] SubediL., TimalsenaS., DuwadiP., ThapaR., PaudelA., ParajuliK., Antioxidant activity and phenol and flavonoid contents of eight medicinal plants from Western Nepal, J. Tradit. Chin.Med., vol.34, pp. 584-590, 2014.

[10]Dongmei Liu, Jiwen Sheng, Zhijian Li, Huimin Qi, Yanlong Sun, Yu Duan, Weifen Zhang, Antioxidant activity of polysaccharide fractions extracted from Athyriummultidentatum(Doll.)Ching, InternationalJournalof Biological Macromolecules, vol.56, pp. 1-5, 2013.

[11] Servili M, Selvaggini R, Esposto S, Taticchi A, Montedoro G,Morozzi G, Health and sensory properties of virgin olive oil hydrophilic phenols: agronomic and technological aspects of production that affect their occurrence in the oil, J Chromatogr A, vol.1054, pp. 113-127, 2004. 